\title{
Recombinant antigen-based immuno-slot blot method for serodiagnosis of syphilis
}

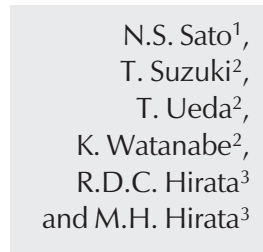

Correspondence

N.S. Sato

Seção de Sorologia

Divisão de Biologia Médica

Instituto Adolfo Lutz

Av. Dr. Arnaldo, 355, 10 andar

01246-902 São Paulo, SP

Brasil

Fax: +55-11-3085-3505

E-mail: neuzasat@uol.com.br

Presented at the 99th General Meeting of the American Society for Microbiology, Chicago, IL, USA,

May 30-June 3, 1999.

Research supported by CAPES, JICA.

Publication supported by FAPESP.

$\ldots \ldots \ldots \ldots \ldots \ldots \ldots$

Received July 15, 2003

Accepted April 5, 2004

$\ldots \ldots \ldots \ldots \ldots \ldots \ldots$.

\author{
'Seção de Sorologia, Divisão de Biologia Médica, Instituto Adolfo Lutz, \\ São Paulo, SP, Brasil \\ ${ }^{2}$ Department of Chemistry and Biotechnology, Graduate School of Engineering, \\ University of Tokyo, Tokyo, Japan \\ ${ }^{3}$ Departamento de Análises Clínicas e Toxicológicas, Faculdade de Ciências \\ Farmacêuticas, Universidade de São Paulo, São Paulo, SP, Brasil
}

\section{Abstract}

Three recombinant antigens of Treponema pallidum Nichols strain were fused with GST, cloned and expressed in Escherichia coli, resulting in high levels of GST-rTp47 and GST-rTp17 expression, and supplementation with arginine tRNA for the AGR codon was needed to obtain GST-rTp15 overexpression. Purified fusion protein yields were 1.9, 1.7 and $5.3 \mathrm{mg} / \mathrm{l}$ of cell culture for GST-rTp47, GST-rTp17 and GST-rTp15, respectively. The identities of the antigens obtained were confirmed by automated DNA sequencing using ABI Prism 310 and peptide mapping by Finningan LC/MS. These recombinant antigens were evaluated by immuno-slot blot techniques applied to 137 serum samples from patients with a clinical and laboratory diagnosis of syphilis (61 samples), from healthy blood donors (50 samples), individuals with sexually transmitted disease other than syphilis (3 samples), and from individuals with other spirochetal diseases such as Lyme disease (20 samples) and leptospirosis (3 samples). The assay had sensitivity of $95.1 \%$ (95\% CI, 86.1 to $98.7 \%$ ) and a specificity of $94.7 \%$ (95\% CI, 87.0 to $98.7 \%$ ); a stronger reactivity was observed with fraction rTp17. The immunoreactivity results showed that fusion recombinant antigens based-immuno-slot blot techniques are suitable for use in diagnostic assays for syphilis.

Treponema pallidum subsp. pallidum is the etiological agent of syphilis, a sexually transmitted disease (STD), and serology is essential for screening and for its diagnosis. However, a major problem for the improvement of treponemal tests is the limited availability of $T$. pallidum, a spirochetal bacterium that cannot be readily cultured in vitro (1). For most research and diagnostic pur-
Key words

- Treponema pallidum

- Cloning

- Recombinant antigen

- Syphilis

- GST poses this bacterium is propagated by intratesticular infection of rabbits, but only small quantities of bacteria are obtained by this procedure. Also, the complexity of the antigenic structures of T. pallidum (2), contamination with rabbit testicular components and high cost have restricted the use of purified specific proteins. Recombinant DNA technology is particularly attractive for obtaining 


\section{T. pallidum antigen.}

The major T. pallidum antigen identified in studies of humoral ontogeny of experimental syphilis (3) and by Western blot analysis has a molecular mass of $47 \mathrm{kDa}, 17$ $\mathrm{kDa}$ and $15 \mathrm{kDa}(4,5)$. All three antigens have been characterized as highly immunogenic membrane lipoproteins (6-10) and as pathogen-specific antigens $(6,10)$. Their DNA sequence has also been reported $(6,9,11)$.

Based on published sequence data for Tp47, Tp17 and Tp15 $(6,9,11)$ these three proteins of T. pallidum subsp. pallidum have been cloned in fusion with GST and expressed in Escherichia coli (12). In the Western blot assay the purified recombinant antigens showed reactivity specifically against the treponemal fraction of the fusion protein, as no serum sample reacted with purified GST (13). In the present study we evaluated the GST-rTp47, GST-rTp17 and GST-rTp15 antigens in an immuno-slot blot (IB) assay for the diagnosis of syphilis.

For cloning, the DNA fragments of Tp47, Tp17 and Tp15 were prepared by PCR using the chromosomal DNA of T. pallidum subsp. pallidum (Nichols strain) as template. The open reading frame (ORF) encoding the fulllength mature protein was amplified by PCR with a primer designed according to a published sequence, GenBank accession numbers M27493, M74852 and M3941 (6,9,11). The sense primer includes the BamHI site and the antisense primer includes the EcoRI site except for Tp47, which had the BamHI site in both primers. PCR cycling (PerkinElmer 9600, Foster City, CA, USA) conditions were as follows: denaturation at $94^{\circ} \mathrm{C}$ for $5 \mathrm{~min}, 30$ cycles of $94^{\circ} \mathrm{C}$ for $1 \mathrm{~min}, \mathrm{~T}$ annealing for $1 \mathrm{~min}, 72^{\circ} \mathrm{C}$ for $1 \mathrm{~min}$ for polymerization, and a final elongation step at $72^{\circ} \mathrm{C}$ for $10 \mathrm{~min}$. T-annealing was carried out at $49^{\circ}, 59^{\circ}$ and $62^{\circ} \mathrm{C}$ for $\mathrm{Tp} 47, \mathrm{Tp} 17$ and Tp15, respectively. Restriction digestion of vector pGEX4T-2 (Pharmacia) and PCR products and ligation were performed according to manufacturer instructions. E. coli
DH5 $\alpha$ or JM109 were used for cloning. Correct insertion of amplified DNA fragments into the expression vector was confirmed by automatic sequencing using a dye terminator cycle sequencing kit and an Applied Biosystems Prism 310 DNA Analyzer (Foster City, CA, USA).

Recombinant $T$. pallidum antigen was expressed in distinct strains of $E$. coli. The antigens GST-Tp47, GST-Tp17 and GSTTp15 were expressed in E. coli BL-21, DH5 $\alpha$ and BL-21/ArgU218, respectively. Cells were grown at $37^{\circ} \mathrm{C}$ in $\mathrm{LB}$ medium containing $200 \mu \mathrm{g} / \mathrm{ml}$ ampicillin for $E$. coli DH5 $\alpha$ or BL-21, and in medium containing $200 \mu \mathrm{g} / \mathrm{ml}$ ampicillin and $30 \mu \mathrm{g} / \mathrm{ml}$ kanamycin for BL-21/ArgU218. Cell growth was monitored by absorbance at $600 \mathrm{~nm}$ and recombinant protein expression was induced by adding isopropyl $\beta$ - $\mathrm{D}$ thiogalactopyranoside to a final concentration of $0.1 \mathrm{mM}$ when absorbance at $600 \mathrm{~nm}$ reached about 0.600 . Four hours after induction, cells were harvested and disrupted by sonication in icecold 0.15 M PBS, pH 7.2, with $1 \mathrm{mM}$ PMSF, $5 \mathrm{mM}$ DTT, and $1 \%$ Triton X-100 and centrifuged at $10,000 \mathrm{~g}$ for $20 \mathrm{~min}$ at $4^{\circ} \mathrm{C}$. The soluble fraction of GST fusion proteins was purified using glutathione (Sigma).

Peptide mapping was performed by Finningan LC/MS (Thermoquest) using a purified rTp antigen digested with peptidases. Briefly, purified rTp was obtained by cleavage with thrombin, with each $10 \mu \mathrm{g}$ protein being incubated for $16 \mathrm{~h}$ at $25^{\circ} \mathrm{C}$ with $0.1 \mathrm{U}$ thrombin in $50 \mathrm{mM}$ Tris- $\mathrm{HCl}, \mathrm{pH} 7.5$, $150 \mathrm{mM} \mathrm{NaCl}, 2.5 \mathrm{~mm} \mathrm{CaCl}_{2}$, and $5 \%$ glycerol before or after elution from the matrix. After electrophoretic separation and protein staining, the rTp band was cut out from the gel and SDS was removed from the gel slices with $0.2 \mathrm{M}$ ammonium bicarbonate in $50 \%$ acetonitrile. The gel slices were lyophilized and soaked in a buffer containing $0.2 \mathrm{M}$ ammonium bicarbonate, $0.2 \%$ Tween 20 and an appropriate amount of TPCKTrypsin (Pierce, Rockford, IL, USA). After 
15 -h incubation at $30^{\circ} \mathrm{C}$, peptides were extracted from the gel with $0.1 \%$ TFA in $60 \%$ acetonitrile, dried and re-dissolved in $0.1 \%$ formic acid for LC/MS analysis.

Recombinant proteins were analyzed by 12\% SDS-PAGE stained with Coomassie brilliant blue R-250 and by Western blotting with serum from patients with secondary syphilis (1:100) as primary antibody and immunodetection was performed using horseradish peroxidase (HRP)-labeled secondary antibody and 4-chloro-1-naphthol.

The diagnostic evaluation of purified recombinant fusion antigen was performed by IB-rTp using 137 serum samples, 61 of which were from patients with a clinical and or laboratory diagnosis of syphilis. For IB, the antigen was dotted onto a nitrocellulose membrane using a Bio-Dot SF apparatus (Bio-Rad, Hercules, CA, USA), and the membrane was cut into $3.0-$ or $4.5-\mathrm{mm}$ wide strips. After titration, the following amounts of each antigen were dotted: 750, 440, 1,670, and $1,250 \mathrm{ng} / \mathrm{mm}^{2}$ for GST-rTp47, GSTrTp17, GST-rTp15, and purified GST, respectively. All strips were prepared with GST, which was used as control for specificity. All reaction steps were performed with shaking at room temperature. Nonspecific binding sites were blocked with sample buffer (0.15 M PBS, pH 7.3, 5\% skim milk, and $0.1 \%$ Tween 20 ) for $15 \mathrm{~min}$, and the strips were washed once with washing buffer $(0.15 \mathrm{M}$ PBS, $\mathrm{pH} 7.3$, and $0.2 \%$ Tween 20). A human serum sample diluted 1:100 or goat anti-GST serum diluted 1:2,000 (Pharmacia, Uppsala, Sweden), used as control to identify GST bands, was added to the strips and incubated for $1 \mathrm{~h}$. After washing three times for 10 min each, the strips were incubated for $60 \mathrm{~min}$ with a second antibody conjugated with HRP. Goat anti-human IgGHRP (Sigma) at 1:2,000 dilution in sample buffer was used as the second antibody. Strips were washed as before, and the color was developed by adding $0.6 \mathrm{mg} / \mathrm{ml} 4$-chloro1-naphthol (Sigma) in 0.15 M PBS, pH 7.3, with $0.02 \% \mathrm{H}_{2} \mathrm{O}_{2}$. The final reaction was stopped by washing the strips with distilled $\mathrm{H}_{2} \mathrm{O}$. When at least one band of GST-rTp was present, with reactivity equal to or stronger than the weakly positive control, the result was considered to be positive.

The serum samples were divided into five groups. Group 1 consisted of 36 serum samples from patients in different clinical stages of syphilis ( 5 with secondary syphilis, 14 in the recent latent phase, and 17 in the late latent phase). These samples were from the control serum panel of the Department of Serology, Adolfo Lutz Institute, São Paulo, SP, Brazil, obtained from patients who were referred to the Department of Sanitary Dermatology of the School of Public Health, University of São Paulo, São Paulo, SP, Brazil. Blood samples were drawn from patients attending the outpatient clinic for sexually transmitted diseases, who had a diagnosis of syphilis based on clinical, epidemiological and laboratory data. Group 2 consisted of 25 serum samples with positive results for treponemal and cardiolipin tests referred to the Adolfo Lutz Institute for syphilis serology. Group 3 consisted of 50 normal serum samples collected from healthy blood donors at the Blood Bank Center of São Paulo, General Hospital of the School of Medicine, USP. All donors were routinely screened for syphilis, and all of them presented negative results. Group 4 consisted of 3 serum samples from patients of the Department of Sanitary Dermatology, School of Public Health, University of São Paulo, with a clinical diagnosis of an STD other than syphilis, who were seronegative for syphilis. Group 5 consisted of serum samples from patients with other spirochete infections. There were 3 samples from patients with leptospiral infection referred to the Adolfo Lutz Institute for leptospiral serology, and presenting seroconversion in agglutination and ELISA tests. Also, 20 serum samples from patients with a clinical and laboratory diagnosis of Lyme disease, kindly provided 
Figure 1. Purity and reactivity of recombinant antigens. Analysis of affinity chromatography-purified GST-rTP fusion protein by (A) Coomassie brilliant blue (CBB)-stained SDS-PAGE and (B) Western blotting performed on serum samples from human syphilis patients and developed with horseradish peroxidase and 4-chloro-1-naphtol (4Cl1N). $\mathrm{M}=$ marker protein, with molecular mass in $\mathrm{kDa}$ shown on the left. Lane 1, GST-rTp47; lane 2, GST-rTp17; lane 3, GSTrTp15. Representative recombinant Treponema pallidum antigen-based immuno-slot blot (IB-rTp) reaction (C) of recombinant GST-rTp antigens and purified GST with goat anti-GST (lane 1); serum from patient with syphilis (lanes 2-11); serum from a healthy negative donor (lanes 12-16); serum from patients with STD other than syphilis (lanes 17-19); serum from patients with leptospirosis (lanes 20-22), and serum from patients with Lyme disease (lanes 23-27). by Dr. Natalino Yoshinari (FM-USP), were also tested. After blood clotting, serum samples were separated by centrifugation and aliquots were stored at $-20^{\circ} \mathrm{C}$. Control serum samples consisted of pooled samples from patients with syphilis, one sample strongly positive, one sample weakly positive in conventional treponemal tests, and one sample pooled serum samples from healthy donors with negative serological results for syphilis. All serum samples were tested by Veneral Disease Research Laboratory (Behring, Marburg, Germany) and fluorescent treponemal antibody-absorption (FTA-Abs, Wama Diagnostica, São Carlos, SP, Brazil) procedures performed according to manufacturer instructions.

GST-rTp47 and GST-rTp17 were successfully expressed in E. coli, whereas GSTrTp15 was poorly expressed in E. coli. However, this effect was completely reversed by supplementation with arginine tRNA for the AGR codon. The ORF of Tp15 contains 4 minor arginine codons including one tandem AGG-AGG codon, which was identified as the inhibitory factor for the

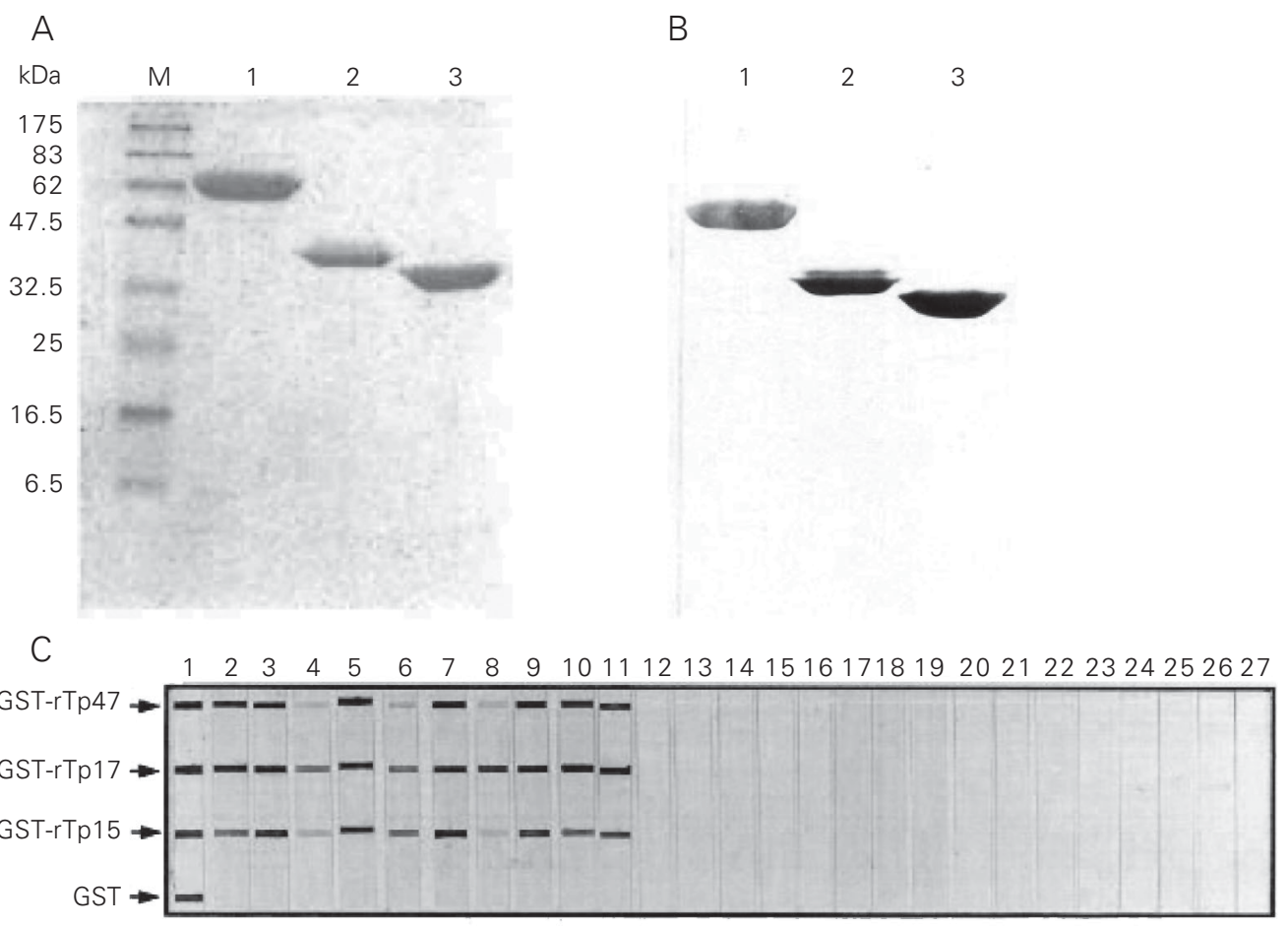

expression of GST-rTp15 (12, and Sato NS, Suzuki T, Hirata RDC, Hirata MH and Watanabe $\mathrm{K}$, unpublished results). Codon use is critical for overexpression of heterologous protein by $E$. coli . It has been reported that the arginine tRNA for rare AGR codons is a limiting factor in the bacterial expression of several heterologous genes due to its infrequent use in E. coli (14). Replacing the rare codons with more commonly used codons and the co-expression of rare tRNA increased significantly the expression level of GST-rTp15 (12, and Sato NS, Suzuki T, Hirata RDC, Hirata MH and Watanabe K, unpublished results). The GST-rTp17 protein was expressed in doublet and the upper band was also specific, as shown in Figure $1 \mathrm{~B}$, reacting with human syphilis serum. This protein with higher molecular weight resulted from amber suppression at stop codon TAG, since this antigen was expressed in E. coli DH5 $\alpha$, which carries the supE44genotype responsible for this translational event.

All three clones showed an exuberant expression of recombinant protein; how-

B 
ever, a large portion of GST-rTp47 and GST-rTp15 remained in the insoluble fraction. The yield of purified fusion protein recovered from the soluble fraction was 1.9, 7.0 and $3.5 \mathrm{mg} / \mathrm{l}$ for GST-rTp47, GSTrTp17 and GST-rTp15, respectively. Experiments are currently being conduced to improve the solubilization of expressed protein in order to obtain better yields. The use of sarkosyl buffer increased GST-rTp47 solubility, although its effects on binding to affinity beads should be evaluated (data not shown). Sarkosyl buffer added to the pellet after sonication has been reported to result in a significant increase in solubility without a drastic denaturing effect on protein (15).

The purity of recombinant antigen expressed in E. coli is especially important because antibodies against contaminating $E$. coli protein may cause false-positive reactions. The expression of recombinant antigens in fusion with GST allowed an efficient one-step purification by glutathione Sepharose 4B affinity chromatography. All three antigens showed high purity and reactivity with a serum sample from a patient with diagnosis of syphilis (Figure 1A,B).

The antigenicity of purified recombinant fusion protein was evaluated by IB. Results of IB and classical serological tests for syphilis for 137 clinical samples are presented in Table 1. A representative reaction profile is shown in Figure 1C. Reaction with anti-GST (lane 1) in IB displays the positions of GSTrTp and GST.

Almost all of the samples from patients with syphilis reacted with the rTp17 antigen, and at a higher intensity compared to the other antigens. Some samples demonstrated weak or no reactivity against the $47-\mathrm{kDa}$ and/ or 15-kDa antigens (Figure 1C, lanes 2-11). A stronger reactivity of the $17-\mathrm{kDa}$ antigen was also reported by other investigators (16). Three patients with latent syphilis presented a negative result in both IB-rTP and FTA-Abs tests (Table 1).

Samples from patients with other spiro- chete infections showed no cross-reaction with samples from patients with leptospirosis. However, 4 samples from patients with Lyme disease had some faint and weak background reaction with at least one recombinant antigen and/or purified GST. These four samples showed a nonspecific background reaction in the FTA-Abs test also. The false-positive reaction in serum samples from patients with Lyme disease has been reported in ELISA for syphilis using a native whole antigen (17). When using whole cells as antigen, it may be speculated that crossreaction may be due to the lipid component present in the native antigen, since the major antigens are lipoproteins in both T. pallidum and Borrelia burgdorferi (18-20). The background reaction observed in the IB assay was unexpected, since the recombinant antigen has no lipid modification. The specificity of these $T$. pallidum recombinant antigens cross-reacting with antibodies can be further evaluated by Western blotting techniques using whole cell antigens of B. burgdorferi.

Serum samples from blood donors and from patients with STD other than syphilis were all negative by IB-rTp, indicating a

Table 1. Results of IB-rTp and other serological tests for syphilis (FTA-Abs and VDRL) for different clinical serum samples analyzed.

\begin{tabular}{|c|c|c|c|c|c|c|}
\hline \multirow[t]{2}{*}{ Sample type } & \multicolumn{2}{|c|}{ IB-rTp } & \multicolumn{2}{|c|}{ FTA-Abs } & \multicolumn{2}{|c|}{ VDRL } \\
\hline & + & - & + & - & + & - \\
\hline \multicolumn{7}{|l|}{ Syphilis } \\
\hline Secondary (5) & 5 & 0 & 5 & 0 & 5 & 0 \\
\hline Early latent (14) & 13 & 1 & 13 & 1 & 9 & 5 \\
\hline Late latent (17) & 15 & 2 & 15 & 2 & 10 & 7 \\
\hline Unknown (25) & 25 & 0 & 25 & 0 & 25 & 0 \\
\hline Normal individual (50) & 0 & 50 & 0 & 50 & 0 & 50 \\
\hline STD other than syphilis (3) & 0 & 3 & 0 & 3 & 0 & 3 \\
\hline \multicolumn{7}{|l|}{ Spirochetal disease } \\
\hline Leptospirosis (3) & 0 & 3 & 0 & 3 & 0 & 3 \\
\hline Lyme disease $(20)^{1}$ & 0 & 16 & 0 & 16 & $N D$ & ND \\
\hline
\end{tabular}

${ }^{1}$ Four samples showed a faint background reaction in IB-rTP with at least one of the recombinant antigens and presented a nonspecific background reaction in the FTAAbs test. FTA-Abs = fluorescent treponemal antibody-absorption; IB-rTp = recombinant Treponema pallidum antigen-based immuno-slot blot assay; STD = sexually transmitted disease; $\mathrm{VDRL}=$ Veneral Disease Research Laboratory; $\mathrm{ND}=$ not done. The number of samples is given in parentheses. 
highly specific reactivity of these tested recombinant antigens. Similar results were obtained by Western blotting (13).

Analysis by the IB technique showed that none of the samples studied reacted with GST, indicating that the reaction to the treponemal portion of the fusion protein is specific. Although the treponemal portion (rTp) might be purified after cleavage of fusion protein (GST-rTp) by treatment with thrombin, this step could be excluded from the protocol in view of the specificity observed in the present study.

The advantage of the IB test is that it is of lower cost and is less cumbersome to perform, since it does not require SDS-PAGE or electrophoretic transfer to nitrocellulose membranes. Furthermore, the amount of antigen dotted on the nitrocellulose membrane is minimal when compared with SDSPAGE/Western blotting. The IB-rTp membrane for the detection of anti-T. pallidum $\mathrm{IgG}$ antibodies was dotted with recombinant protein amounts as low as $23,13,50$, and 38 ng per strip for GST-rTp47, GST-rTp17, GST-rTp15, and GST, respectively. Fusion recombinant antigen-based IB techniques are suitable for use in diagnostic assays for syphilis. Further studies on the application of recombinant antigen-based IB for detection of different anti-T. pallidum antibody isotypes (IgM and $\operatorname{IgA}$ ), as well as ELISA for screening tests are now being conducted.

\section{Acknowledgments}

The authors are grateful to Dr. Zila Rosa Belém (Biolab, São Paulo, SP, Brazil) for providing T. pallidum Nichols strain, to Dr. Kazunori Yamada, $\mathrm{PhD}$ (Molecular Medicine Laboratory, Yokohama Researcher Center, Mitsubishi Chemical Corporation, Japan), for donating BL-21/pArgU218, to Dr. Luiz Jorge Fagundes from the Health Center "Geraldo de Paula Souza", School of Public Health, USP, SP, for providing serum samples from patients with a clinical diagnosis of syphilis and STD other than syphilis, to Dr. Amadeo Saez Alquézar from Blood Bank Center of São Paulo, SP, for seronegative samples from blood donors, and to Dr. Natalino Yoshinari, Department of Reumathology, Faculty of Medicine, USP, SP, for providing serum samples from patients with a diagnosis of Lyme disease. We are grateful to Dr. Chie Takemoto and Akio Inouye, University of Tokyo, for helpful technical advice, and also to the group of the Laboratory of Serology, Adolfo Lutz Institute, São Paulo, Brazil, for collaboration. Neuza Satomi Sato was a participant in the Training Program for Researchers of Japanese Descent sponsored by the Japan International Cooperation Agency (JICA), from April 1997 to April 1998. She thanks Dr. Elsa M. Mamizuka and Dr. Mirthes Ueda for encouragement while participating in this program.

\section{References}

1. Fieldsteel AH, Cox DL \& Moeckli RA (1981). Cultivation of virulent Treponema pallidum in tissue culture. Infection and Immunity, 32: 908-915.

2. Norris SJ \& Stell S (1994). Antigenic complexity of Treponemas pallidum: antigenicity and surface localization of major polypeptides. Journal of Immunology, 133: 2686-2692.

3. Hanff PA, Bishop NH, Miller JN \& Lovett MA (1983). Humoral response in experimental syphilis to polypeptides of Treponema pallidum. Journal of Immunology, 131: 1973-1977.

4. Byrne RE, Laska S, Bell M, Larson D, Philips J \& Todd J (1992). Evaluation of a Treponema pallidum Western immunoblot assay as a confirmatory test for syphilis. Journal of Clinical Microbiology, 30: 115-122.

5. Hanff PA, Fehninger TE, Miller JN \& Lovett MA (1982). Humoral immune response in human syphilis to polypeptides of Treponema pallidum. Journal of Immunology, 129: 1287-1291.

6. Akins DR, Purcell BK, Mitra MM, Norgard MV \& Radolf JD (1993) Lipid modification of the 17-kilodalton membrane immunogen of Treponema pallidum determines macrophage activation as well as amphiphilicity. Infection and Immunity, 61: 1202-1210.

7. Chamberlain NR, Brandt ME, Erwuin AL, Radolf JD \& Norgard MV (1989). Major integral membrane protein immunogens of Tre- 
ponema pallidum are proteolipids. Infection and Immunity, 57: 2872-2877.

8. Norgard MV, Chamberlain NR, Swancutt MA \& Goldberg MS (1986). Cloning and expression of the major 47-kilodalton surface immunogen of Treponema pallidum in Escherichia coli. Infection and Immunity, 54: 500-506.

9. Weigel LM, Brandt ME \& Norgard MV (1992). Analysis of the Nterminal region of the 47-kilodalton integral membrane lipoprotein of Treponema pallidum. Infection and Immunity, 60: 1568-1576.

10. Centurion-Lara A, Arrool T, Castilho R, Shaffer JM, Castro C, van Voorhis WC \& Lukehart SA (1997). Conservation of the 15-kilodalton lipoprotein among Treponema pallidum subspecies and strains and other pathogenic treponemes: genetic and antigenic analyses. Infection and Immunity, 65: 1440-1444.

11. Purcell BK, Chamberlain NR, Goldberg MS, Andrews LP, Robinson EJ, Norgard MV \& Radolf JD (1989). Molecular cloning and characterization of the 15-kilodalton major immunogen of Treponema pallidum. Infection and Immunity, 57: 3708-3714

12. Sato NS (1999). Clonagem, expressão e caracterização de antígenos recombinantes do Treponema pallidum com potencial imunodiagnóstico. Doctoral thesis, Faculty of Pharmaceutical Sciences, University of São Paulo, São Paulo, SP, Brazil.

13. Sato NS, Hirata MH, Hirata RDC, Zerbini LCMS, Silveira EPR, Melo CS \& Ueda M (1999). Analysis of Treponema pallidum recombinant antigens for diagnosis of syphilis by Western blotting technique. Revista do Instituto de Medicina Tropical de São Paulo, 41: 115-
118.

14. Brinkmann U, Mattes RE \& Buckel P (1989). High-level expression of recombinant genes in Escherichia coli is dependent on the availability of the dnaY gene product. Gene, 85: 91-114.

15. Grieco F, Hay JM \& Hull RI (1992). An improved procedure for the purification of protein fused with glutathione S-transferase. BioTechniques, 13: 856-858.

16. Fujimura K, Ise N, Ueno E, Hori T, Fujii N \& Okada M (1997). Reactivity of recombinant Treponema pallidum ( $r-T p$ ) antigens with anti-Tp antibodies in human syphilitic sera evaluated by ELISA. Journal of Clinical and Laboratory Analysis, 11: 315-322.

17. Lefevre JC, Bertrand MA, Bauriaud R \& Lareng MB (1992). False positive reactions occurring with Captia Syphilis G EIA in sera from patients with Lyme disease. Genitourinary Medicine, 68: 142 (letter).

18. Radolf JD, Chamberlain NL, Clausell A \& Norgard MV (1988). Identification and localization of integral membrane proteins of virulent Treponema pallidum subsp. pallidum by phase partitioning with nonionic detergent Triton X-114. Infection and Immunity, 56: 490-498.

19. Belisle JT, Brandt ME, Radolf JD \& Norgard MV (1994). Fatty acids of Treponema pallidum and Borrelia burgdorferi lipoproteins. Journal of Bacteriology, 176: 2151-2157.

20. Brandt ME, Riley BS, Radolf JD \& Norgard MV (1990). Immunogenic integral membrane proteins of Borrelia burgdorferi are lipoproteins. Infection and Immunity, 58: 983-991. 